\title{
DESARROLLO DE UNA TAXONOMÍA DE ACTIVIDADES DE PREVENCIÓN Y PROMOCIÓN DE LA SALUD EN ATENCIÓN PRIMARIA
}

Cristina Lozano Ochoa y Belén Marín Palacios

Escuela de Enfermería de la Universidad de La Rioja 


\section{RESUMEN}

\section{Referencia del documento de práctica clínica basada en la evidencia:}

Pumar-Méndez MJ, Lopez-Dicastillo O, Hernantes N, Iriarte-Roteta A, Belintxon $M$, Garcia-Iriarte A, et al. Development of a taxonomy of activities in health prevention and promotion for primary care. J Nurs Manag. 2020;00:1-13.

Disponible en: https://doi.org/10.1111/ jonm.12980

\section{Introducción.}

La prevención y la promoción de la salud están incluidas en la cartera de servicios de los sistemas de salud más sólidos. Su provisión y desarrollo se consideran una pieza clave para la protección, el mantenimiento y la mejora de salud de la población, así como para la sostenibilidad del sistema sanitario. Sin embargo, la implementación de la prevención y promoción de salud sigue siendo insuficiente, variable y depende de la implicación de los profesionales. Esto se hace evidente en los profesionales de enfermería, que a pesar de ser un grupo altamente competente y con actitud positiva ante este tipo de intervenciones, su actividad se limita a la educación sanitaria en los estilos de vida.

La falta de implementación de estas actividades ha sido aceptada y normalizada. De hecho, las omisiones en prevención y promoción de salud rara vez se consideran como un problema para el paciente, a pesar de que este hecho ya ha sido evidenciado. Un estudio realizado en 27 países de la OCDE encontró que los daños más relevantes en atención primaria fueron fallos y demoras en la detección y tratamiento de enfermedades crónicas. Estos errores conducen al deterioro de la salud y a un aumento de la probabilidad de hospitalización en los pacientes.

El origen de la falta de conciencia sobre las omisiones en prevención y promoción de la salud es multifactorial. La atención primaria puede ser el escenario idóneo para desarrollar un nuevo enfoque para estandarizar la prevención y promoción de la salud y en concreto las omisiones de éstas actividades cuyo problema sigue sin resolverse.

\section{Objetivo}

Desarrollar la primera taxonomía de actividades en prevención y promoción de la salud para atención primaria. 


\section{Métodos, fuentes de datos}

En este estudio se llevo a cabo el desarrollo de una taxonomía, definiendo la misma como un catálogo de actividades que deben realizar los profesionales de la salud para desarrollar plenamente la prevención y promoción de la salud en atención primaria. Para ello se realizó una revisión de estas actividades, para identificar áreas de mejora en la prevención y promoción de la salud incluyendo prácticas desentendidas o errores de omisión. La revisión se realizó por un equipo de investigadores compuesto por académicos especializados en prevención y promoción de salud, seguridad del paciente, atención comunitaria y gestión de salud y profesionales asistenciales en atención primaria. Tras revisar las taxonomías y clasificaciones existentes diseñadas para atención primaria y verificar que ninguna de ellas había iniciado la catalogación de la prevención y promoción de la salud, el equipo de investigación decidió desarrollar una taxonomía desde cero, utilizando el análisis documental.

El marco teórico utilizado para el desarrollo de la taxonomía fue la Carta de Otawa, la cual guió el muestreo, la selección y el análisis de documentos.

Para identificar los documentos, se llevaron a cabo cinco búsquedas bibliográficas en MEDLINE, cada una con una combinación de palabras claves específicas para localizar registros relacionados con un área de acción específica de la Carta de Otawa. Las búsquedas se limitaron a inglés y castellano y entre enero de 2007 y enero de 2018. Además se realizó la técnica de bola de nieve. Una vez seleccionados, los documentos se analizaron siguiendo el enfoque de marco. Éste ofrece un proceso analítico que comprende cinco etapas: familiarización, identificación de un marco temático, gráficos de indexación y mapeo e interpretación.

\section{Resultados}

Las búsquedas bibliográficas realizadas mostraron 4.321 documentos. Tras aplicar los criterios de inclusión y exclusión y la lectura de los títulos, resúmenes y texto completo se incluyeron un total de 62 documentos. El análisis de los documentos siguiendo el marco temático dio como resultado una lista inicial de 47 códigos. La versión de la Taxonomía final de actividades en prevención y promoción de la salud en atención primaria (TaxoPromo) está disponible en castellano, inglés y portugués.

TaxoPromo incluye 43 actividades de prevención y promoción de salud agrupadas en ocho categorías.

- Planificación

- Análisis situacional 
- Desarrollar capacidad organizacional para la promoción de salud

- Generar conciencia/opinión pública para la promoción de salud

- Abogacía para la promoción de salud

- Desarrollo de redes para la promoción de salud

- Desarrollo de colaboraciones para la promoción de la salud

- Estrategias de intervención en promoción de salud

\section{COMENTARIO}

La promoción de salud fue definida en la Carta de Ottawa en el año 1986 como el proceso que permite que las personas ejerzan un control sobre los determinantes de la salud, mejorando su bienestar. Sin embargo a día de hoy, las actividades de prevención y promoción de salud deberían tener más relevancia en el sistema sanitario.

La responsabilidad de realizar intervenciones de prevención y promoción de la salud dentro del sistema sanitario está asignada a atención primaria. Además, el profesional de enfermería es uno de los principales agentes implicados en el desarrollo de dichas competencias. Sin embargo, la evidencia muestra que la participación de enfermería en la evaluación de prevención y promoción de la salud, la planificación estratégica y el desarrollo de políticas públicas saludables es especialmente escasa (1).

Las causas de su falta de desarrollo son múltiples: factores individuales (formación, creencias, motivación, autoconfianza), del equipo (apoyos, perfil de la comunidad) o institucionales (objetivos de las gerencias, modelo biomédico, falta de visibilidad de resultados a corto plazo, falta de demanda por parte de la población) (2).

La herramienta TaxoPromo se convierte en la primera taxonomía creada para la evaluación sistemática de prácticas de prevención y promoción de la salud en atención primaria.

Tal y como se ha mencionado la herramienta fue creada en base a un marco teórico, en concreto la Carta de Ottawa, además de revisar otras herramientas similares previo a su elaboración. Es importante destacar la explicación detallada de cada categoría, su definición y cada uno de sus componentes.

Dicha herramienta puede ser muy útil para detectar que actividades se llevan a cabo, así como las omisiones y detectar las posibles áreas de mejora.

Los profesionales de enfermería deben desarrollar las competencias de prevención y promoción de salud, sin embargo la evidencia muestra que no se 
hace de forma global y está centrada principalmente en la educación para la salud.

TaxoPromo puede ser utilizada en cada organización de atención primaria involucrando a todos los miembros del equipo para identificar áreas específicas de mejora. Los datos pueden servir para diseñar planes u objetivos anuales de implementación y prevención y promoción de salud, identificar acciones para integrar las actividades de forma sistemática y permitir la evaluación comparativa.

La obtención de datos sobre prevención y promoción de la salud en la práctica de atención primaria puede realizarse de forma novedosa tras la elaboración de TaxoPromo.

Para concluir la utilización de una herramienta como Taxopromo puede servir a enfermería para clarificar y expandir sus competencias tanto en la prevención como en la promoción, y no sólo centrar su interés educación para la salud, consiguiendo alcanzar una mejora en el bienestar de la población.

\section{REFERENCIAS}

1. Iriarte A, López-Dicastillo O, Mujika A, Ruiz Zaldibar C, Hernantes N, Bermejo E y Pumar-Méndez MJ. Nurses'role in health promotion and prevention: a critical interpretive synthesis. 2020;

2. Rubio-Valera M, Pons-Vigues M, Martínez-Andrés M, Moreno-Peral $P$, Berenguera $A$ y Fernández A. Barriers and facilitators for the implementation of primary prevention and health promotion activities in primary care: a synthesis through meta-etnography, PLoS one. 2014; 9(2):e89554. 